\title{
PERFIL DE FLAVONOIDES E AVALIAÇÃO DO POTENCIAL ANTIOXIDANTE E CITOTÓXICO DE Bauhinia purpurea (Fabaceae) DA REGIÃO AMAZÔNICA
}

\author{
Priscila Moraes dos Santos ${ }^{\mathrm{a}}$, Patrícia Danielle Oliveira de Almeida ${ }^{\mathrm{b}}$, Emerson Silva Lima ${ }^{\mathrm{b}}$, Manoel Odorico de Moraes \\ Patrícia Marçal da Costa ${ }^{\mathrm{c}}$, Assuero Silva Meira ${ }^{\mathrm{c}}$, Cláudia do Ó Pessoa ${ }^{\mathrm{c}}$, Ligia Maria Marino Valente ${ }^{\mathrm{d}}$ e Valdir Florêncio \\ da Veiga Junior ${ }^{\mathrm{a}, *}$

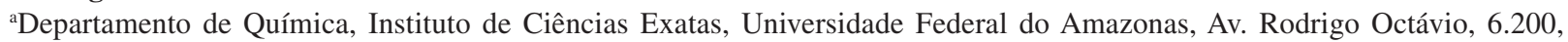 \\ 69077-040 Manaus - AM, Brasil \\ ${ }^{b}$ Faculdade de Ciências Farmacêuticas, Universidade Federal do Amazonas, Rua Alexandre Amorin, Manaus - AM, Brasil \\ 'Universidade Federal do Ceará, Rua Coronel Nunes de Melo, 1127, Fortaleza - CE, Brasil \\ dInstituto de Química, Universidade Federal do Rio de Janeiro, Av. Athos da Silveira Ramos 149, Centro de Tecnologia, Bl. A, \\ 21941-909 Rio de Janeiro - RJ, Brasil
}

Recebido em 24/06/2013; aceito em 27/08/2013; publicado na web em 16/10/2013

\begin{abstract}
FLAVONOID PROFILES AND EVALUATION OF ANTIOXIDANT POTENTIAL AND CYTOTOXICITY OF AMAZONIAN Bauhinia purpurea (Fabaceae). Plants of the Bauhinia species (Fabaceae) are used in the treatment of several infections and diseases, especially diabetes. In this paper, with an aim to contribute to the knowledge of the chemistry and therapeutic potential of the Amazonian species of the genus Bauhinia, flavonoid profiles in the leaves and branches of Bauhinia purpurea collected in Manaus and São Gabriel da Cachoeira/AM were analyzed by HPLC-DAD and ESIMS. Differences in the flavonoid profiles were detected along with the presence of rutin and isoquercitrin in the leaves collected in Manaus. All samples showed antioxidant activity, however, no cytotoxic activity against cell lines MDA-MB435 (breast), HCT-8 (colon), and SF-295 (glioblastoma) could be detected.
\end{abstract}

Keywords: Bauhinia purpurea; rutin; isoquercitrin.

\section{INTRODUÇÃO}

O gênero Bauhinia pertencente à família Fabaceae (subfamília: Cercideae) contém cerca de 300 espécies que são conhecidas popularmente no Brasil como pata-de-vaca pelo formato bilobado de suas folhas. ${ }^{1}$ Elas são usadas na medicina tradicional em diversas partes do mundo por suas propriedades analgésica, antidiabética, antiinflamatória, antimicrobiana, adstrigente, diurética no tratamento de inchaços e tumores estomacais, diarréia e úlcera. ${ }^{2-5} \mathrm{O}$ uso popular mais comumente relatado para esse gênero no Brasil está relacionado à atividade hipoglicemiante, com várias espécies usadas para esse fim, em especial Bauhinia forficata, que é amplamente comercializada em variadas formas farmacêuticas. ${ }^{6,7}$

As espécies de Bauhinia contêm diversas substâncias, como flavonoides livres e glicosilados, terpenoides, esteroides, ácidos fenólicos e alcaloides. ${ }^{6-9}$ De espécimes asiáticos de $B$. purpurea foram isolados além de flavonoides, como kaempferol-3-galactosídio, isoquercitrina, rutina e astragalina, compostos raros da classe oxepinos, bauhiniastatinas, alguns com atividade citotóxica. ${ }^{10-12}$

De um modo geral, as propriedades terapêuticas das espécies, especialmente as atividades antioxidante e hipoglicemiante, têm sido correlacionadas à presença de compostos fenólicos, em especial de flavonoides glicosilados, como a kaempferitrina, vinculada à ação hipoglicemiante da espécie $B$. forficata. ${ }^{13-15}$

O estudo da espécie $B$. purpurea frente à atividade antioxidante vem ganhando destaque no estudo do gênero, já que seus extratos têm exibido potencial intermediário ou equivalente aos padrões geralmente utilizados nos testes para esta atividade. As substâncias fenólicas, atribuídas como as principais responsáveis por essas atividades, tem sido investigadas por meios cromatográficos e espectrométricos, na busca para estabelecer um perfil cromatográfico dentro da espécie. ${ }^{16-19}$

*e-mail: valdirveiga@ufam.edu.br
Na região amazônica, o uso de espécies desse gênero não é diferente, havendo relatos para o tratamento de diabetes nos estados do Acre, Amazonas e Pará. As espécies são encontradas em muitas feiras e comércios a céu aberto, especialmente folhas, para uso como chá..$^{20-22}$

Apesar do grande interesse que essas espécies despertam, há poucos relatos sobre a composição química e o potencial terapêutico das existentes na região amazônica. ${ }^{23-27}$

Neste trabalho, com o objetivo de contribuir para o conhecimento da constituição química e do potencial terapêutico de espécies amazônicas do gênero Bauhinia, foi realizada uma análise inicial a partir da detecção de flavonoides (CLAE e IES-EM) e das atividades antioxidante e citotóxica de folhas e galhos de $B$. purpurea, coletados nas cidades de Manaus e São Gabriel da Cachoeira, estado do Amazonas.

\section{EXPERIMENTAL}

\section{Material vegetal e preparo dos extratos}

A espécie $B$. purpurea teve folhas e galhos coletados na Vila Olímpica de Manaus (FBp1 e GBp1, respectivamente) e folhas no município de São Gabriel da Cachoeira (folhas - FBp2). As exsicatas encontram-se depositadas no Herbário da Universidade Federal do Amazonas, sob os números 7932 e 07657 , respectivamente. O material, cerca de $700 \mathrm{~g}$, foi seco à temperatura ambiente por sete dias, separado, limpo, triturado e extraído por maceração com metanol PA $(\mathrm{MeOH})$. O solvente foi eliminado à pressão reduzida a $40{ }^{\circ} \mathrm{C}$ gerando respectivamente 1,$6 ; 26,8$ e $2,7 \mathrm{~g}$ dos extratos secos, que foram armazenados a $4{ }^{\circ} \mathrm{C}$.

\section{Fracionamento dos extratos}

Foram realizadas partições utilizando $1,0 \mathrm{~g}$ de cada extrato bruto de $B$. purpurea solubilizando-se em $10 \mathrm{~mL}$ de $\mathrm{MeOH}: \mathrm{H}_{2} \mathrm{O}(9: 1)$ e 
particionando sequencialmente com diclorometano PA $\left(\mathrm{CH}_{2} \mathrm{Cl}_{2}\right)$ (4 x $75 \mathrm{~mL}$ ) e acetato de etila PA (AcOEt) $(4$ x $75 \mathrm{~mL})$. As frações AcOEt obtidas foram utilizadas para elaboração do perfil cromatográfico das espécies estudadas através de CCD, CLAE-DAD e EM. ${ }^{28}$ Elas foram identificadas como: FBp1-F e GBp1-F (frações AcOEt provenientes do extrato bruto de folhas e galhos, respectivamente, do espécime coletado em Manaus) e FBp2-F (fração AcOEt proveniente do extrato bruto de folhas do espécime coletado em São Gabriel da Cachoeira).

\section{Cromatografia em camada delgada (CCD)}

Para realização da CCD utilizou-se cromatofolha de gel de sílica $60 \mathrm{~F}_{254}$ (Merck) com espaçamento de $0,5 \mathrm{~cm}$ nos lados e entre as amostras e $0,5 \mathrm{~cm}$ de banda de aplicação das amostras. Nas margens inferior e superior da placa foram demarcados 1 e $0,5 \mathrm{~cm}$, respectivamente, sendo $8 \mathrm{~cm}$ o espaço total percorrido pelas amostras na placa. Com auxílio de seringa Hamilton de $25 \mu \mathrm{L}, 15 \mu \mathrm{L}$ de cada fração na concentração de $50 \mathrm{mg} / \mathrm{mL}$ e dos padrões isoquercitrina (Aldrich), kaempferitrina (isolada das folhas da espécie Uncaria guianensis) ${ }^{29}$ e rutina (Aldrich) na concentração de $1 \mathrm{mg} / \mathrm{mL}$ foram aplicados. Usou-se como fase móvel AcOEt/HCOOH/ $\mathrm{CH}_{3} \mathrm{COOH} / \mathrm{H}_{2} \mathrm{O}$ 100:10:10:27 e, para revelação, borrifamento com NP (difenilboriloxietilamina 1,0\%, em MeOH), seguido de PEG (polietilenoglicol 4000, 5,0\% em EtOH), com observação sob lâmpada de UV a $365 \mathrm{~nm} .{ }^{30}$

\section{Cromatografia líquida de alta eficiência (CLAE-DAD)}

Análise realizada na UFRJ, com equipamento marca PerkinElmer série 200, composto por bomba quaternária, desgaseificador a vácuo, amostrador automático, forno para colunas e detector de arranjo de diodos (DAD) Perkin-Elmer série 200EP, equipado com coluna Lichrocart LiChrospher 100RP18, de dimensões 250 x 4,6 $\mathrm{mm}$, com partículas de $5 \mu \mathrm{m}$ de diâmetro. $\mathrm{O}$ detector foi fixado em uma faixa de aquisição de 200-600 nm, a uma taxa de aquisição de espectro de $88 \mathrm{scans} / \mathrm{s}$. As frações obtidas em AcOEt, assim como os padrões isoquercitrina, rutina e kaempferitrina, foram analisados em modo gradiente com $\mathrm{CH}_{3} \mathrm{CN}$ (solvente $\mathrm{B}$ ) e $\mathrm{H}_{2} \mathrm{O} / \mathrm{HCOOH} \mathrm{pH}=3,0$ (solvente A): 30 min $95-75 \%$ de A; 30-50 min, $75-20 \%$ de A; 50$55 \mathrm{~min}, 20-95 \%$ de $\mathrm{A}$; $55-60 \mathrm{~min} 95 \%$ de $\mathrm{A}$, vazão de $1,0 \mathrm{~mL} / \mathrm{min}$, volume de injeção $20 \mu \mathrm{L}$, temperatura $30{ }^{\circ} \mathrm{C}$, comprimentos de onda de aquisição 265 e $320 \mathrm{~nm}$. Todas as amostras foram analisadas na concentração de $10 \mathrm{mg} / \mathrm{mL}$.

\section{Espectrometria de massas com ionização por eletrospray (IES- EM)}

Utilizou-se equipamento LCQ FleetTM (Thermo Scientific), localizado na UFAM, equipado com fonte de eletrospray, operando no modo negativo, com analisador do tipo ion trap. As amostras foram diluídas em MeOH grau UV e $200 \mu \mathrm{L}$ com concentração de $10 \mu \mathrm{g} /$ $\mathrm{mL}$, foram injetados por meio de inserção direta. Utilizou-se como substâncias de referência rutina e isoquercitrina.

Foi utilizado o programa Xcalibur para obtenção dos espectros na faixa de m/z 100 - 1000, com análise EM/EM e EM3 dos picos relativos aos padrões flavonoídico rutina e isoquercitrina.

\section{Avaliação do potencial antioxidante frente o radical livre DPPH}

Para atividade antioxidante de captura do radical DPPH foi realizado o monitoramento da solução de DPPH (Aldrich), 0,3 mM/mL em $\mathrm{MeOH}$, no aparelho leitor de microplaca (DTX 800, Beckman) em comprimento de onda de $492 \mathrm{~nm}$ até obtenção de absorbância de $1,00( \pm 0,1)$. Na microplaca de 96 poços foram aplicados $270 \mu \mathrm{L}$ da solução hidroalcóolica de DPPH e $30 \mu \mathrm{L}$ da solução de extrato de $B$. purpurea diluído em DMSO nas concentrações iniciais de 50, 100, 250,500 e $1000 \mu \mathrm{g} / \mathrm{mL}$ (sendo estas minimizadas quando apresentavam alta atividade). Para o controle negativo foi utilizado DMSO. Todas as análises foram realizadas em triplicata.

Após 30 min de incubação à temperatura ambiente e na ausência de luz, foi realizada a leitura em $492 \mathrm{~nm} .{ }^{31}$

A substância quercetina foi utilizada como padrão referência e o cálculo da $\mathrm{CI}_{50}$ foi obtido através da seguinte fórmula:

$$
\% \text { inibição }=100-\left(\frac{\text { abs amostra }}{\text { abs controle }}\right) \times 100
$$

\section{Avaliação do potencial antioxidante frente o radical livre A BTS}

A atividade antioxidante frente ao radical livre ABTS constituiu-se de uma solução catiônica de ABTS (7 mM, 50\% de solução de ABTS, $50 \%$ solução de perssulfato de potássio $5 \mathrm{mM}$ ) mantida em temperatura ambiente e com ausência de luz onde, após 12 horas, foi formado o radical $\mathrm{ABTS}^{\bullet+}$. Após a formação do radical foi realizado primeiramente o monitoramento da solução, sendo adicionados na microplaca $270 \mu \mathrm{L}$ da solução hidroalcóolica de ABTS (diluição 1:7 da solução original) mais $30 \mu \mathrm{L}$ de etanol, efetuando-se a leitura em $417 \mathrm{~nm}$ através do leitor de microplaca (DTX 800, Beckman). Ao se obter absorbância de aproximadamente $1,00( \pm 0,1)$, foi realizado o ensaio em triplicata através da adição de $270 \mu \mathrm{L}$ da solução hidroalcóolica de ABTS com $30 \mu \mathrm{L}$ da amostra em diferentes concentrações (concentrações iniciais de 50, 100, 250, 500 e $1000 \mu \mathrm{g} / \mathrm{mL}$ ). Para o controle negativo foi utilizado DMSO em concentração única. Todas as análises foram realizadas em triplicata.

Após incubação de 15 min em temperatura ambiente e na ausência de luz, realizou-se a leitura no leitor de microplaca em $417 \mathrm{~nm} \cdot{ }^{32} \mathrm{~A}$ substância Trolox ${ }^{\circledR}$ (Aldrich) foi utilizada como padrão referência e o cálculo do $\mathrm{CI}_{50}$ foi realizado por meio da mesma equação do ensaio anterior.

\section{Avaliação do potencial antioxidante frente o radical $\mathrm{O}_{2}$.}

O procedimento foi baseado na reação entre NADH (nicotinamida adenina dinucleotídeo), PMS (fenazina metassulfato) e NBT (azul de nitrotetrazólio), onde é formado o ânion radical superóxido. Assim, dois radicais superóxido doam, cada um, um elétron para o NBT, reduzindo e formando a formazam, que é monitorado em um comprimento de onda de $560 \mathrm{~nm}$, em espectrofotômetro UV-Vis modelo DTX 800, Beckman. ${ }^{33}$

Foram preparados inicialmente os reagentes NBT $(250 \mu \mathrm{M})$, NADH $(390 \mu \mathrm{M})$ e PMS $(10 \mu \mathrm{M})$ em tampão Tris-HCl $(16 \mathrm{mM}$, $\mathrm{pH}=8,0)$ e, em seguida, em uma placa de 96 poços foram adicionados: $50 \mu \mathrm{L}$ de solução dos extratos metanólicos de B. purpurea nas concentrações de 10 a $1000 \mu \mathrm{g} / \mathrm{mL}, 100 \mu \mathrm{L}$ de NADH e $100 \mu \mathrm{L}$ de NBT. A absorbância inicial foi obtida a partir da leitura da microplaca a $560 \mathrm{~nm}$. Em seguida foram adicionados $100 \mu \mathrm{L}$ de PMS. Todas as análises foram realizadas em triplicata.

A mistura foi então incubada a $25^{\circ} \mathrm{C}$ por 5 minutos e, logo após, realizada a leitura da absorbância final a $560 \mathrm{~nm} \cdot{ }^{34} \mathrm{~A}$ atividade foi calculada através da seguinte fórmula:

$$
\% \text { inibição }=100-\left(\frac{a b s_{\text {final }} \text { amostra }-a b s_{\text {inicial }} \text { amostra }}{a b s \text { controle }}\right) \times 100
$$




\section{Avaliação do potencial citotóxico em células tumorais}

A determinação do potencial antitumoral das amostras foi verificada através da análise da citotoxidade dos extratos em três linhagens de células tumorais: MDA-MB435 (mama - humano), HCT-8 (cólon humano) e SF-295 (glioblastoma - humano). Para um screening inicial, os extratos foram testados na concentração de $50 \mu \mathrm{g} / \mathrm{mL}$, concentração adotada pelo National Cancer Institute dos Estados Unidos (NCI). ${ }^{35}$

Após adição dos extratos diluídos nas amostras, incubou-se por 72 horas em estufa a $5 \%$ de $\mathrm{CO}_{2}$ a $37{ }^{\circ} \mathrm{C}$. Ao término deste, as mesmas foram centrifugadas e o sobrenadante removido. Em seguida, foram adicionados $150 \mu \mathrm{L}$ da solução de MTT (sal de tetrazólio) e as placas foram incubadas por $3 \mathrm{~h}$. A absorbância foi lida após dissolução do precipitado com $150 \mu \mathrm{L}$ de DMSO puro em espectrofotômetro de placa a $595 \mathrm{~nm} .^{36}$

\section{RESULTADOS}

No esforço de caracterização das substâncias responsáveis pelas propriedades terapêuticas de Bauhinia forficata, em especial aquelas relacionadas à diabetes, foram isolados flavonóis glicosilados ativos, como a kaempferitrina (1) ${ }^{13}$ e descrita a correlação entre misturas de derivados de kaempferol, quercetina, miricetina e isorramnetina a estas propriedades. ${ }^{15}$ Baseado nesses dados, buscamos caracterizar o perfil de flavonoides das amostras amazônicas de B. purpurea por CCD, CLAE-DAD e IES-EM, assim como verificar a presença de kaempferitrina e demais flavonoides já caracterizados no gênero.

\section{Detecção de flavonoides por CCD, CLAE-DAD e IES-EM}

A análise preliminar da presença de compostos polifenólicos nas frações AcOEt provenientes dos extratos metanólicos das folhas e galhos dos dois espécimes de B. purpurea foi feita por CCD, utilizando-se os flavonóis glicosilados kaempferitrina (1), isoquercitrina (2) e rutina (3) como substâncias de referência. Tanto na coloração quanto no $\mathrm{R}_{\mathrm{f}}$, foram observados indícios da presença de rutina e isoquercitrina (material suplementar) nas frações de folhas e galhos de B. purpurea.

A kaempferitrina, Figura 1, flavonoide glicosilado característico da espécie $B$. forficata, não foi observada em nenhuma das amostras de $B$. purpurea, indicando a ausência da mesma nesta espécie.

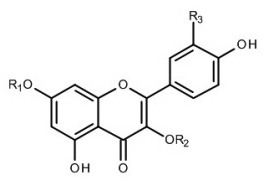

$1-\mathrm{R}_{1}=\mathrm{R}_{2}=\alpha-$ Ramnose, $\mathrm{R}_{3}=\mathrm{H}$

2 - $\mathrm{R}_{1}=\mathrm{H}, \mathrm{R}_{2}=\beta-$ Glicose, $\mathrm{R}_{3}=\mathrm{OH}$

$3-\mathrm{R}_{1}=\mathrm{H}, \mathrm{R}_{2}=\alpha$-Ramnosil $(1 \rightarrow 6) \beta-$ Glicose, $\mathrm{R}_{3}=\mathrm{OH}$

Figura 1. Estrutura dos flavonoides glicosilados presentes em espécies de Bauhinia usados como substâncias de referência: (1) kaempferitrin; (2) isoquercitrina; (3) rutina

Os cromatogramas em CLAE-DAD das frações revelaram diferenças nos perfis flavanoídicos.

Os resultados obtidos em CLAE-DAD evidenciaram a presença dos flavonoides rutina e isoquercitrina nas frações dos extratos obtidos das folhas coletadas em Manaus e São Gabriel da Cachoeira. O flavonoide kaempferitrina, característico da espécie Bauhinia forficata, não foi verificado nas frações analisadas. No perfil cromatográfico da fração relativa aos galhos de B. purpurea, coletados em Manaus, os resultados não permitiram a conclusão sobre a presença das substâncias de referência, em função da complexidade das misturas e da baixa concentração de seus componentes (Figura 2C).

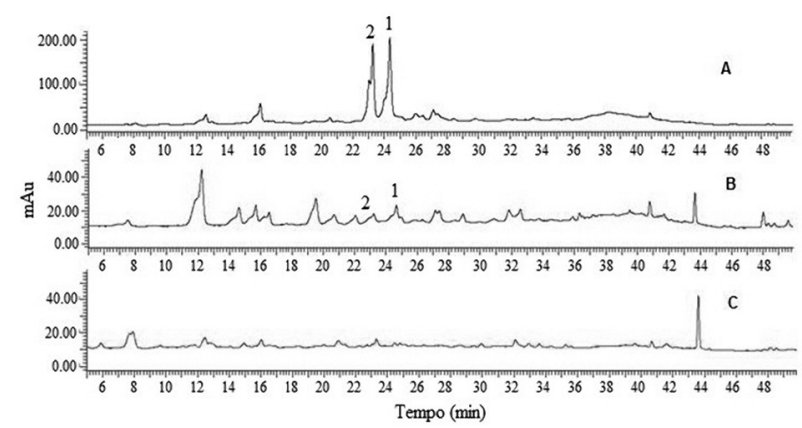

Figura 2. Cromatogramas em CLAE-DAD a $265 \mathrm{~nm}$ das frações flavonoídicas dos extratos de folhas coletadas em Manaus (FBpl-F) (A) e em São Gabriel da Cachoeira $(F B p 2-F)(\boldsymbol{B})$, com a presença dos padrões de isoquercitrina (1) e rutina (2); e dos galhos coletados em Manaus (GBp1-F) (C)

Na sequência, as amostras de $B$. pupurea foram submetidas à análise por EM, Figura 3, utilizando-se rutina e isoquercitrina como padrões de referência.

Nos espectros das amostras analisadas (Figura 3) pode-se observar a diferença no perfil composicional entre os locais de coleta, assim como entre as diferentes partes da planta.

Nesta análise, por meio dos espectros obtidos no modo negativo, as duas substâncias foram detectadas em todas as amostras através da presença dos íons relativos à [M-H] em m/z 609 e 463 respectivamente (Figura 3). Sua detecção nas frações dos extratos de folhas do espécime de São Gabriel da Cachoeira e nos galhos do espécime de Manaus em concentrações bem menores que as obtidas nas folhas do espécime de Manaus confirma a maior sensibilidade da técnica em relação à CLAE-DAD, onde essas substâncias não haviam sido detectadas.

Para confirmação da presença destes dois flavonoides nas amostras, realizou-se a análise por $\mathrm{EM}^{\mathrm{n}}$ dessas moléculas desprotonadas, comparando os resultados com aqueles obtidos dos espectros das substâncias padrão, nas mesmas condições de análise (Material Suplementar). Os resultados mostraram um padrão de fragmentação característico para as duas substâncias, com perda das unidades de açúcar $(m / z$ 609/301 e $m / z$ 463/301) e a fragmentação das respectivas agliconas gerando o íon ${ }^{1,2} \mathrm{~A}^{-}(\mathrm{m} / \mathrm{z}, 301 / 179)$, ambas em concordância com a literatura ${ }^{37-43}$ (Material suplementar).

Esse é o primeiro relato da presença de rutina em $B$. purpurea, a qual já havia sido detectada em outras espécies do gênero Bauhinia como B. vahlii, B. tomentosa, B. splendens e B. retusa. ${ }^{44-48}$ Isoquercitrina foi isolada anteriormente de espécime asiático de $B$. purpurea, e também em B. tomentosa e B. retusa. ${ }^{5,47-49}$

Indícios de outros flavonoides glicosilados também podem ser observados nos espectros, como em $\mathrm{m} / \mathrm{z} 477$, presente nas amostras de B. purpurea de Manaus (FBp1-F e GBp1-F), e m/z 623 em FBp1-F e FBp2-F, ambos característicos de derivados de isoramnetina, isoramnetina 3-O- $\beta$-D-glucosídeo e isoramnetina 3-O- $\beta$-rutinosídeo, respectivamente. Essa hipótese é fundamentada na identificação dos mesmos em espécies do gênero, como em $B$. purpurea e $B$. blakeana. ${ }^{50-53}$

Os íons em $m / z, 289$ e 577 em GBp1-F podem estar relacionados à presença da epicatequina e duas unidades da mesma, chamada epicatequina-epicatequina, também já identificada anteriormente em espécies de Bauhinia, como B. championii Benth. e B. aurea. ${ }^{54-56}$

\section{Potencial antioxidante e citotóxico}

O resultado do potencial antioxidante dos extratos brutos dos dois espécimes amazônicos de $B$. purpurea contra três radicais livres é mostrado na Tabela 1 . 

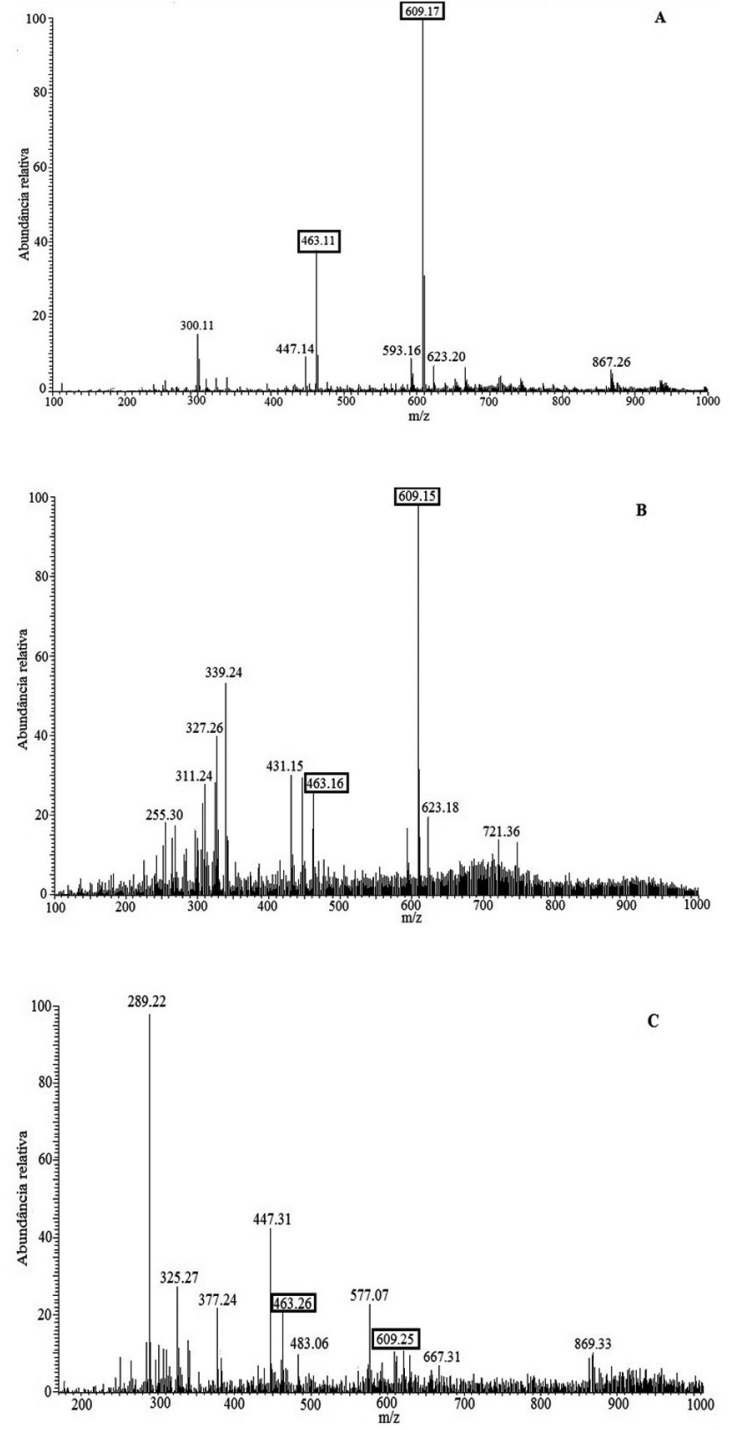

Figura 3. Espectros de massas das amostras de B. purpurea: frações flavonoídicas dos extratos das folhas coletadas em Manaus - FBp1-F (A); folhas coletadas em São Gabriel da Cachoeira - FBp2-F (B); galhos coletados em Manaus - GBp1-F (C), com assinalamento dos íons em $\mathrm{m} / z, 609$, relativo à $[\mathrm{M}-\mathrm{H}]^{-}$do flavonoide rutina e em $\mathrm{m} / \mathrm{z} 463$, relativo à $[\mathrm{M}-\mathrm{H}]^{-}$do flavonoide isoquercitrina

Tabela 1. Avaliação da atividade antioxidante $\left(\mathrm{CI}_{50} \mathrm{em} \mu \mathrm{g} / \mathrm{mL}\right)$ dos extratos metanólicos de folhas e galhos de B. purpurea coletada em Manaus (FBp1 e GBp1 respectivamente) e de folhas da espécie coletada em São Gabriel da Cachoeira $(\mathrm{FBp} 2)$ frente a radicais livres

\begin{tabular}{cccc}
\hline Amostra & DPPH $(\mu \mathrm{g} / \mathrm{mL})$ & ABTS $(\mu \mathrm{g} / \mathrm{mL})$ & $\mathrm{O}_{2}^{-\bullet}(\mu \mathrm{g} / \mathrm{mL})$ \\
\hline GBp1 & $38,29 \pm 1,21$ & $22,13 \pm 1,44$ & $36,75 \pm 1,12$ \\
FBp1 & $195,80 \pm 5,66$ & $45,76 \pm 8,10$ & $694,11 \pm 13,66$ \\
FBp2 & $17,23 \pm 2,07$ & $42,68 \pm 5,56$ & $343,20 \pm 15,90$ \\
Padrão & $2,57 \pm 0,10$ & $6,99 \pm 0,08$ & $11,67 \pm 0,57$ \\
& Quercetina & Trolox & Ácido gálico \\
\hline
\end{tabular}

As maiores atividades antioxidantes dos extratos são frentes aos radicais estáveis DPPH e ABTS.+ do que com o radical superóxido, radical o qual está relacionado a reações de estresse oxidativo, gerados durante o metabolismo como produtos finais de reações enzimáticas ou celulares, sendo considerado como o modelo que mais se aproxima das reações oxidativas biológicas. Tais resultados podem estar relacionados à possibilidade da presença de grupos fenólicos, como epicatequinas, detectados na Figura 2 anteriormente. ${ }^{57,58}$

No teste superóxido, os resultados indicam uma atividade baixa entre os extratos das folhas coletadas em locais diferentes (FBp1 e FBp2). Porém, frente o radical $\mathrm{ABTS}^{+}{ }^{+}$são observados resultados similares para os mesmos.

De forma interessante, os resultados mais consistentes entre os três métodos foram obtidos para o extrato dos galhos do espécime coletado em Manaus (GBp1), revelando um potencial antioxidante para este extrato.

Tal atividade antioxidante obtida para as amostras referentes às folhas contrasta com o perfil flavonoídico encontrado em $B$. purpurea, onde foram detectadas substâncias derivadas de quercetina. Outro aspecto é a diferença na atividade apresentada pela FBp1 e FBp2, onde FBp2 apresenta um índice antioxidante bem menor que FBp1, o que pode estar relacionado à diferença no perfil flavonoídico entre essas amostras, onde FBp2 apresentou mais substâncias fenólicas, de acordo com os resultados das análises em CCD, CLAE e IES-EM realizados.

Os baixos valores apresentados pelo extrato de galhos, GBp1, pode estar relacionado à diferente composição química majoritária nesta parte específica da planta quando comparado com a amostra relativa às folhas, como observado nos espectros de massas, onde são constatados mais picos característicos de compostos fenólicos.

As diferentes técnicas utilizadas com os extratos indicam o comportamento dos mesmos quanto ao seu caráter antioxidante. No teste superóxido, os resultados apresentados são superiores aos testes DPPH e ABTS. Uma possível explicação reside no mecanismo de ação, uma vez que para as análises frente um radical superóxido, espécies como flavanonas, di-hidroflavonóis e flavanóis, assim como derivados destes com substituição em C-6 e C-3, não são capazes de apresentar atividade, sendo essas as principais classes encontradas no gênero, e por isso apresentarem tal baixa atividade..$^{57}$

Os resultados da porcentagem de inibição dos extratos de $B$. purpurea para as diferentes linhagens tumorais testadas encontramse na Tabela 2.

Tabela 2. Percentual de inibição para extratos metanólicos de B. purpurea contra três linhagens de células tumorais: MDA-MB435 (melanoma- humano), HCT-8 (cólon - humano) e SF-295 (glioblastoma - humano)

\begin{tabular}{cccc}
\hline & HCT-8 & MDA/MB-435 & SF-295 \\
\cline { 2 - 4 } & Média \pm DPM & Média \pm DPM & Média \pm DPM \\
\hline FBp2 & $39,55 \pm 2,76 \%$ & $17,77 \pm 3,75 \%$ & $29,75 \pm 0,96 \%$ \\
FBp1 & $28,19 \pm 2,39 \%$ & $1,48 \pm 5,97 \%$ & $25,67 \pm 0,96 \%$ \\
GBp1 & $33,56 \pm 1,19 \%$ & $5,85 \pm 7,70 \%$ & $26,17 \pm 2,95 \%$ \\
\hline
\end{tabular}

É possível observar que nenhum dos extratos apresentou taxa de inibição maior que $40 \%$ contra as linhagens de células testadas, mostrando, portanto, baixa citotoxidade. Entretanto, já foram descritas na literatura atividades inibitórias contra células tumorais a partir de substâncias isoladas de B. purpurea, coletadas na Tailândia células cancerígenas (carcinoma de boca e mama) ${ }^{49}$ o que confronta tais resultados obtidos com o extrato bruto dessa espécie local. Este efeito pode estar associado ao fato do uso do próprio extrato bruto e não de substâncias isoladas ou até mesmo uma composição química diferente nesta espécie amazônica, comparada à asiática relatada na literatura, o que oferece novos caminhos para a pesquisa fitoquímica neste gênero. 


\section{CONCLUSÃO}

O perfil de flavonoides em CLAE-DAD das folhas de dois espécimes de $B$. purpurea da região amazônica, coletados em diferentes localidades, apresentou diferenças relacionadas tanto ao relativo local de coleta quanto às partes das plantas, indicando distinção na composição das amostras estudadas.

A presença dos flavonóis glicosilados rutina e isoquercitrina foi detectada em todas as amostras de folhas e galhos analisadas por $\mathrm{EM}^{\mathrm{n}}$, já a kaempferitrina não foi identificada em nenhuma amostra.

A atividade antioxidante dos extratos brutos variou em relação às amostras e aos métodos utilizados, com destaque para as taxas de inibição obtidas com amostras relativas aos galhos de B. purpurea de Manaus e folhas da mesma espécie da localidade de São Gabriel da Cachoeira.

A diferença da composição química entre as B. purpurea dos diferentes locais podem estar relacionados à maior atividade da FBp2-F, já que a presença de compostos fenólicos nessa amostra é aparentemente maior que em FBp1-F, como visualizado nos resultados de EM.

Nenhum dos extratos de B. purpurea apresentou atividade citotóxica contra as linhagens de células tumorais testadas.

\section{MATERIAL SUPLEMENTAR}

As Figuras 1S à 4S estão disponíveis em http://quimicanova.sbq. org.br, em arquivo pdf, com acesso livre.

\section{AGRADECIMENTOS}

Os autores agradecem o apoio financeiro e tecnológico da FUNCAP, CNPq, FAPEAM e CAPES.

\section{REFERÊNCIAS}

1. Silva, K. L.; Cechinel-Filho, V.; Quim. Nova 2002, 25, 449.

2. Gonzalez-Mujica, F.; Motta, N.; Becerra, A.; Phytother. Res. 1998, 12, 291.

3. Carvalho, J. C. T.; Santos, L. S.; Viana, E. P.; De Almeida, S. S. M. S.; Meconato, E.; Rodrigues, M.; Ferreira, L. R.; Van de Kamp, A.; Pharm. Biol. 1999, 37, 281.

4. Willain Filho, A.; Breviglieri, E.; Cechinel-Filho, V.; Santos, A. R. S.; J. Pharm. Pharmacol. 1997, 49, 823.

5. Kumar, T.; Chandrashekar, K. S.; Res. J. Med. Plant 2011, 5, 420.

6. Nogueira, A. C. O.; Sabino, C. V. S.; Revista Fitos 2012, 7, 77.

7. Cechinel Filho, V.; Phytother. Res. 2009, 23, 1347.

8. Mali, R. G.; Mahajan, S. G.; Mehta, A. A.; Pharmacogn. Rev. 2007, 1, 314.

9. González-Mujica, F.; Em Phytochemicals - Bioactivities and Impact on Health; InTech: Iraj Rasooli, 2011.

10. Vijayakumari, K.; Siddhuraju, P.; Janardhanan, K. L.; J. Sci. Food Agric. 1997, 73, 279 .

11. Ramachandran, R.; Joshi, B. C.; Curr. Sci. 1967, 36, 574

12. Abd-El-Wahab, S. M.; Wassel, G. M.; Ammar, N. M.; Hanna, T.; Herba Hungarica 1987, 26, 27.

13. De Sousa, E.; Zanatta, L.; Seifriz, I.; Creezynski-Pasa, T. B.; Pizzolatti, M. G.; Szpoganiez, B.; Silva, F.; J. Nat. Prod. 2004, 67, 829.

14. Da Cunha, A. M.; Menon, S.; Menon, R.; Couto, A. G.; Bürger, C.; Biavatti, M. W.; Phytomedicine 2010, 17, 37.

15. Ferreres, F.; Gil-Izquierdo, A.; Vinholes, J.; Silva, S. T.; Valentão, P.; Andrade, P. B.; Food Chem. 2012, 134, 898.

16. Zakaria, Z. A.; Loo Y. W.; Abdul Rahman, N. I.; Abdul Ayub, A. H.; Sulaiman, M. R.; Hanan Kumar, G.; Med. Princ. Pract. 2007, 16, 443.

17. Yadava, R. N.; Tripathi, P.; Fitoterapia 2000, 71, 88.
18. Annegowda, H. V.; Mordi, M. N.; Ramanathan, S.; Hamdan, M. R.; Mansor, S. M.; Food Anal. Methods 2012, 5, 226.

19. Zakaria, Z. A.; Rofiee, M. S.; Teh, L. K.; Salleh, M. Z.; Sulaiman, M. R.; Somchit, M. N.; African J. Biotech., 2011, 10, 65.

20. Ming, L. C.; Plantas medicinais na reserva extrativista Chico Mendes - uma visão etnobotânica; Editora UNESP: São Paulo, 2006.

21. Borrás, M. R. L.; Plantas da Amzônia: medicinais ou mágicas? Plantas comercializadas no Mercado Municipal Adolpho Lisboa; Editora Valer: Manaus, 2003.

22. Barbosa, W. L. R.; Etnofarmácia: fitoterapia popular e ciência farmacêutica; Núcleo de Meio Ambiente: Belém- Pará, 2009.

23. Laux, D. O.; Stefani, G. M.; Gottlieb, O. R.; Phytochemistry 1985, 24, 1081.

24. Sauvainb, M.; Bourdyb, G.; Callapaa, J.; Bergeronc, S.; Rojasa, I.; Bravod, J. A.; Balderramad, L.; Ortize, B.; Gimenezf, A.; Deharob, E.; J. Ethnopharm. 2000, 69, 127.

25. Behrens, M. D.; Tappin, M. R. R.; Favoreto, R.; Silva, V. P.; Nakamura, M. J.; Barbosa, A. P.; Sousa, L. A.; Siani, A. C.; Revista Fitos 2006, 1, 58.

26. Silva, E. M.; Souza, J. N. S.; Rogezc, H.; Reesb, J. F.; Larondellea, Y.; Food Chem. 2007, 101, 1012.

27. da Silva Góis, R. W.; de Sousa, L. M.; Santiago, G. M.; Romero, N. R.; Lemos, T. L.; Arriaga, A. M.; Braz-Filho, R.; Parasitol. Res. 2013, in press.

28. Engel, I. C.; Ferreira, R. A.; Rev. Bras. Farm. 2008, 18, 258.

29. Valente, L. M. M.; Bizarri, C. H.; Liechocki, S.; Barboza, R. S.; Almeida, M. B. S.; Benevides, P. J. C.; Magalhães, A.; Siani, A. C.; J. Braz. Chem. Soc. 2009, 20, 1041.

30. Wagner, H.; Bladt, S.; Plant Drug Analysis - A Thin Layer Cromatography Atlas; Second Edition, Springer, 1996.

31. Molyneux, P.; Songklanakarin J. Sci. Technol. 2004, 26, 211.

32. Re, R.; Pellegrini, N.; Proteggente, A.; Pannala, A.; Yang, M.; RiceEvans, C.; Free Radic. Biol. Med. 1999, 26, 1231.

33. Ewing, J. F.; Janero, D. R.; Anal. Biochem. 1995, 232, 243.

34. Öztürk, M.; Aydogmus-Öztürk, F.; Duru, M. E.; Topçu, G.; Food Chem. 2007, 103, 623.

35. Skehan, P.; Storeng, R.; Scudiero, D.; Monks, A.; McMahon, J.; Vistica, D.; Warren, J. T.; Bodesh, H.; Kenney, S.; Boyd, M. R.; J. Natl. Cancer Inst. 1990, 82, 1107.

36. Mossman, T.; J. Immunol. Methods 1983, 65, 55.

37. Dubber, M-J.; Sewram, V.; Mshicileli, N.; Shephard, G. S.; Kanfer, I.; J. Pharm. Biomed. Anal. 2005, 37, 723

38. Silva, S.; Matias, A. A.; Nunes, A.; Duarte, C.; Coelho, A. V.; Bronze, M. R.; Ciência Téc. Vitiv. 2005, 20, 17.

39. Güvenalp, Z.; Demirezer, L. O.; Turk. J. Chem. 2005, 29, 163.

40. Garrett, R.; Romanos, M. T. V.; Borges, R. M.; Santos, M. G.; Rocha, L.; Silva, A. J. R.; Rev. Bras. Farmacogn. 2012, 22, 306.

41. Lin, L.; Harnly, J. M.; Food Chem. 2010, 120, 319.

42. Del Rio, D.; Stewart, A. J.; Mullen, W.; Burns, J.; Lean, M. E. J.; Brighenti, F.; Crozier, A.; J. Agric. Food Chem. 2004, 52, 2807.

43. Koolen, H. H. F.; Soares, E. R.; Silva, F. M. A.; Souza, A. Q. L.; Rodrigues Filho, E.; de Souza, A. D. L.; Rev. Bras. Farmacogn. 2012, $22,189$.

44. Sultana, S.; Ilyas, M.; Mohammed, K.; Shaida, W.A.; J. Indian Chem. Soc. 1985, 62, 337.

45. Tiwari, K. P.; Masood, M.; Rathore, Y. K. S.; Proceedings of the National Academy of Sciences, India Section A: Physical Sciences 1978, $48,183$.

46. Cechinel Filho, V.; Breviglieri, E.; Willain Filho, A.; Santos, A. R. S.; Rev. Bras. Farm. 1995, 76, 115.

47. Row, L.; Ramachandra, L.; Viswanadham, N.; Proc. Indian. Acad. Sci. 1954, 39, 240.

48. Subramanian, S. S.; Nair, A. G. R.; Indian J. Chem. 1963, 1, 450. 
49 Boonphong, S. S.; Puangsombat, P. P.; Baramee, A. A.; Mahidol, C. C.; Ruchirawat, S. S.; Kittakoop, P. P.; J. Nat. Prod. 2007, 70, 795.

50. Gutzeit, D.; Wray, V.; Winterhalter, P.; Jerz, G.; Chromatographia 2007, $65,1$.

51. Spilková, J.; Húbik, J.; Biologische Wirkungen von Flavonoiden. II Pharmazie in unserer Zeit 1992, 21,174.

52. Sinha, K. N.; Singh, M.; Bioscience Discovery 2013, 4, 20.

53. Zakaria, H.; Simpson, K.; Brown, P. R.; Krotulovic, A.; J. Chromatography 1979, 176, 109
54. Xu, W.; Zheng, H.; Hong, Z.; Tang, X.; The Chinese Journal of Modern Applied Pharmacy 2009, 9, 763.

55. Shang, X.; Li, S.; Wang, S.; Yang, Y.; Shi, J.; Zhongguo Zhong Yao Za Zhi 2007, 32, 815 .

56. De Souza, L. M.; Cipriani, T. R.; Iacomini, M.; Gorin, P. A.; Sassaki, G. L.; J Pharm Biomed. Anal. 2008, 47, 59.

57. Vargas, F. S.; Dissertação de Mestrado, Universidade Federal do Amazonas, Brasil, 2008.

58. Cai, Y.; Luo, Q.; Sun, M.; Corke, H.; Life Sci. 2004, 74, 2157. 


\section{PERFIL DE FLAVONOIDES E AVALIAÇÃO DO POTENCIAL ANTIOXIDANTE E CITOTÓXICO DE Bauhinia purpurea (Fabaceae) DA REGIÃO AMAZÔNICA}

Priscila Moraes dos Santos ${ }^{a}$, Patrícia Danielle Oliveira de Almeida ${ }^{b}$, Emerson Silva Lima ${ }^{\mathrm{b}}$, Manoel Odorico de Moraes ${ }^{\mathrm{c}}$, Patrícia Marçal da Costa ${ }^{\mathrm{c}}$, Assuero Silva Meira ${ }^{\mathrm{c}}$, Cláudia do Ó Pessoa ${ }^{\mathrm{c}}$, Ligia Maria Marino Valente ${ }^{\mathrm{d}}$ e Valdir Florêncio da Veiga Junior ${ }^{\mathrm{a}, *}$

${ }^{a}$ Departamento de Química, Instituto de Ciências Exatas, Universidade Federal do Amazonas, Av. Rodrigo Octávio, 6.200, 69077-040 Manaus - AM, Brasil

${ }^{b}$ Faculdade de Ciências Farmacêuticas, Universidade Federal do Amazonas, Rua Alexandre Amorin, Manaus - AM, Brasil. 'Universidade Federal do Ceará, Rua Coronel Nunes de Melo, 1127, Fortaleza - CE, Brasil

dInstituto de Química, Universidade Federal do Rio de Janeiro, Av. Athos da Silveira Ramos 149, Centro de Tecnologia, Bl. A, 21941-909 Rio de Janeiro - RJ, Brasil

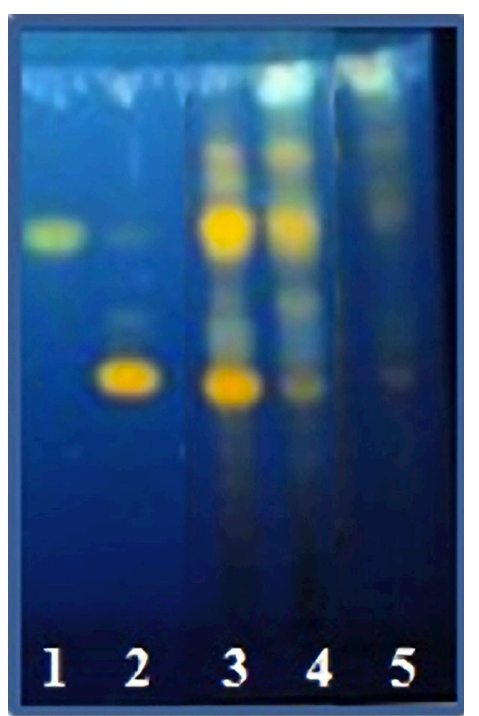

Figura 1S. Placa cromatográfica em UV-365 nm após borrifar com NP-PEG, onde: 1) kaempferitrina, 2) rutina, 3) GBp1-F, 4) FBp1-F, 5) FBp2-F

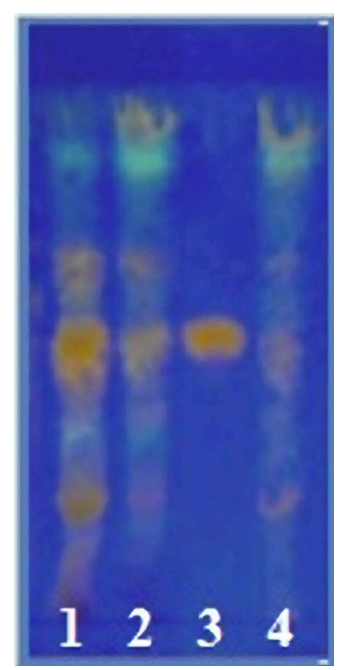

Figura 2S. Placa cromatográfica em UV-365 nm após borrifar com NP-PEG, onde: 1) FBp1-F, 2) GBp1-F, 3) isoquercitrina, 4) FBp2-F

*e-mail: valdirveiga@ufam.edu.br
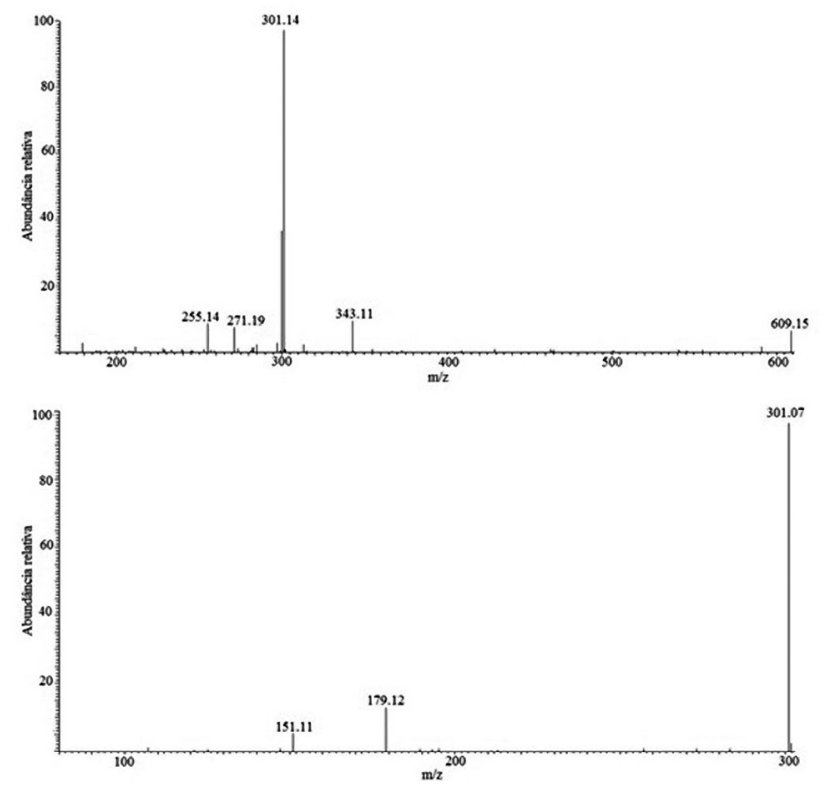

Figura 3S. Espectro de EM/EM do íon m/z 609 e EM/EM do íon $301 \mathrm{em} \mathrm{B.}$ purpurea, relativo ao padrão rutina
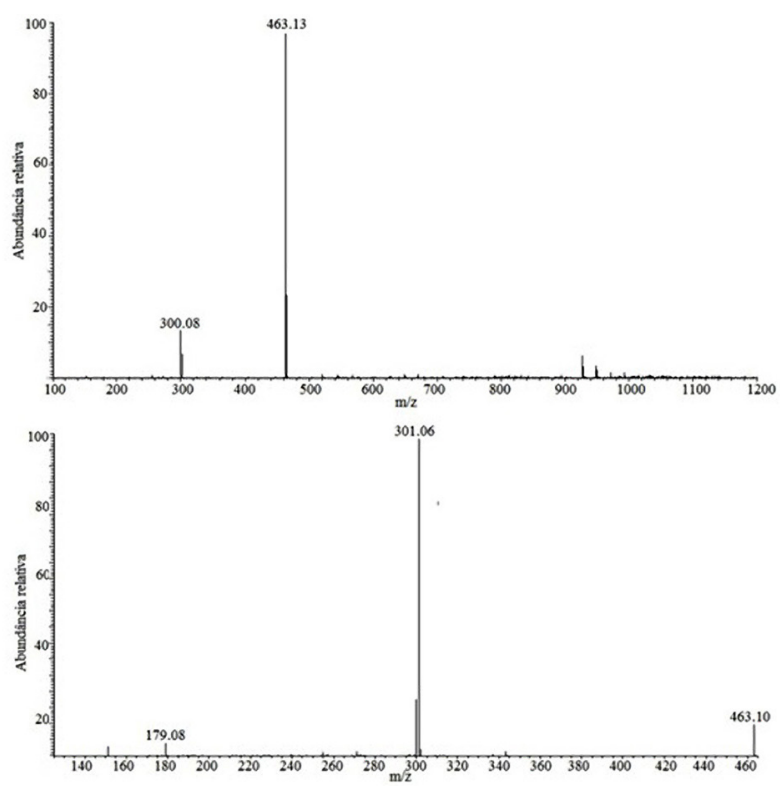

Figura 4S. Espectro de EM e EM/EM do ion $\mathrm{m} / 2463 \mathrm{em}$ B. purpurea, relativo ao padrão isoquercitrina 